\title{
Optical and Microcantilever-Based Sensors for Real-Time In Situ Characterization of High-Level Waste (81924)
}

\section{Research Objective}

Fundamental research is being conducted to develop sensors for cesium and strontium that can be used in real-time to characterize high-level waste (HLW) process streams. Two fundamentally different approaches are being pursued, having in common the dependence on highly selective molecular recognition agents. In one approach, an array of chemically selective sensors with sensitive fluorescent probes to signal the presence of the constituent of interest will be coupled to fiber optics for remote analytical applications. The second approach employs sensitive microcantilever sensors that have been demonstrated to have unprecedented sensitivity in solution for $\mathrm{Cs}^{+}$and $\mathrm{CrO}_{4}{ }^{-}$. Selectivity in microcantilever-based sensors is achieved by modifying the surface of a gold-coated cantilever with a monolayer coating of an alkanethiol derivative of the molecular recognition agent. The approaches are complementary since fiber optic sensors can be deployed in the highly alkaline environment of HLW, but a method of immobilizing a fluorescent molecular recognition agent in a polymer film or bead on the surface of the optical fiber has yet to be demonstrated. The microcantilever-based sensors function by converting molecular complexation into surface stress, and they have been demonstrated to have the requisite sensitivity. However, a method of protecting Si or SiN microcantilever sensors in the highly alkaline environment of HLW while maintaining high selectivity remains to be demonstrated. The fundamental technology for fiber optic and cantilever sensors has been developed by our collaborators David Walt and Thomas Thundat, respectively, and the goal of this project is to adapt molecular recognition chemistry to the methods already being employed. To develop molecular recognition agents for $\mathrm{Cs}^{+}$and $\mathrm{Sr}(\mathrm{II})$ with rapidly established equilibria needed for real-time analysis, we will focus on calixarene-crown ethers as a platform. Sensors for alkali metal ions, hydroxide, and temperature will be part of the array of sensor elements that will be demonstrated in this program for both the cantilever and fiber optic sensor approaches.

\section{Research Progress and Implications}

This report summarizes the research as of June, 2003, following 20 months of research in a three year project. Specific goals in this program are to develop a selective complexation agent for $\mathrm{Sr}$ (II) based on calixarene crown ether chemistry, and then adapt this chemistry to fluorescence sensors and cantilever sensors. In addition to utilizing previously developed $\mathrm{Cs}^{+}$ selective 1,3-alt-calix[4]arene-benzocrown-6 compounds having a cyanoanthracene fluorophore group to develop fiber optic sensors, we want to develop additional fluorophores that are more tolerant of the polymer matrix which immobilizes the complex. Appropriate reagents for making cantilevers specific for $\mathrm{Sr}$ and $\mathrm{Cs}$ as well as the chemistry to apply a passivating layer for microcantilever sensors that is tolerant of strongly alkaline solutions need further investigation.

Arrays of sensors based on imaging fiber optics are prepared by attaching the indicating chemistries to the fiber by one of three general approaches: polymer sensing layers, discrete polymer sensor elements on an imaging fiber, or microwell arrays with the reagent attached to microspheres. In the latter method, a random distribution of the microspheres is introduced into the optical fiber array wells. A robust method for encoding and registering the microspheres after assembly is required, and the Tufts team has developed a computer algorithm that enables the different microsphere types to be recognized and positionally registered. The next step in the research will be to attach the indicating chemistries developed at ORNL onto microspheres and 
to test the responses of the individual microspheres after immobilization. Polystyrene-based beads appear to be the most attractive choice for highly alkaline solution. The stability of optical fibers in alkali is a potential problem, and one possibility is to use PMMA fibers or to coat glass imaging fibers with a material that prevents the glass from being attacked by the alkali.

Work is in progress to develop coatings to make microcantilever sensors that are selective for $\mathrm{Sr}(\mathrm{II})$. It appears from the literature that an amide-ether combination is one element that can impart selectivity. We have previously demonstrated that the thiophenol derivative, shown below, is selective for $\mathrm{Ca}^{2+}$, but a monolayer of this compound

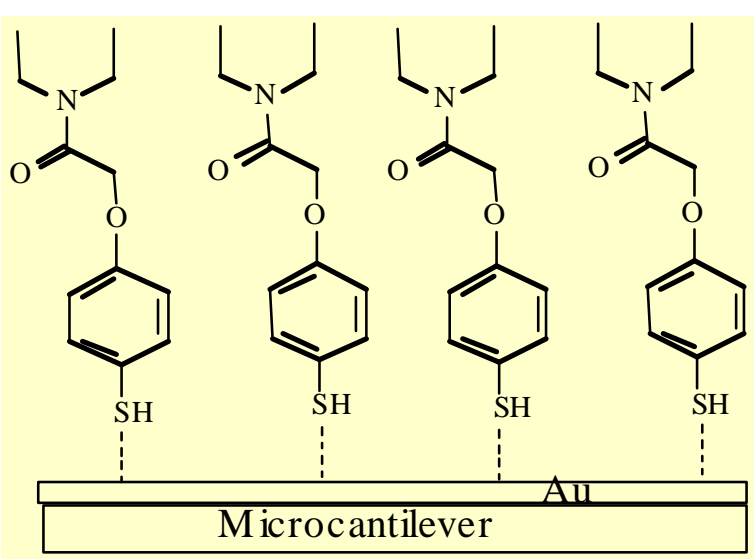

gives little selectivity for $\mathrm{Sr}(\mathrm{II})$.

The synthesis of the undecanethioacetate derivative shown to the right is in progress, and it will be tested when it becomes available. Upon reacting with the gold surface, the acetate group will be hydrolyzed to acetic acid, leaving the alkanethiol sorbed to the gold.

The synthesis and a study of the fluorescence of a

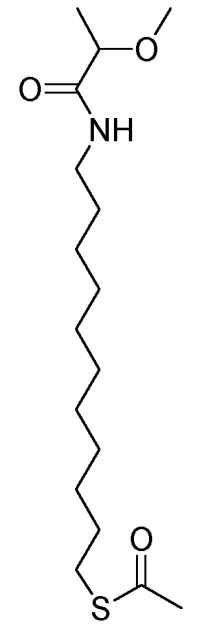

calix[4]arene based $\mathrm{Sr}^{2+}$ probe has been nearly completed. The molecule was prepared by alkylation of the lower rim of calix[4]arene in the cone conformation, and other derivatives of (aminocarbonylmethoxy)calix[4] arene have been shown by others to complex $\mathrm{Sr}^{2+}$.
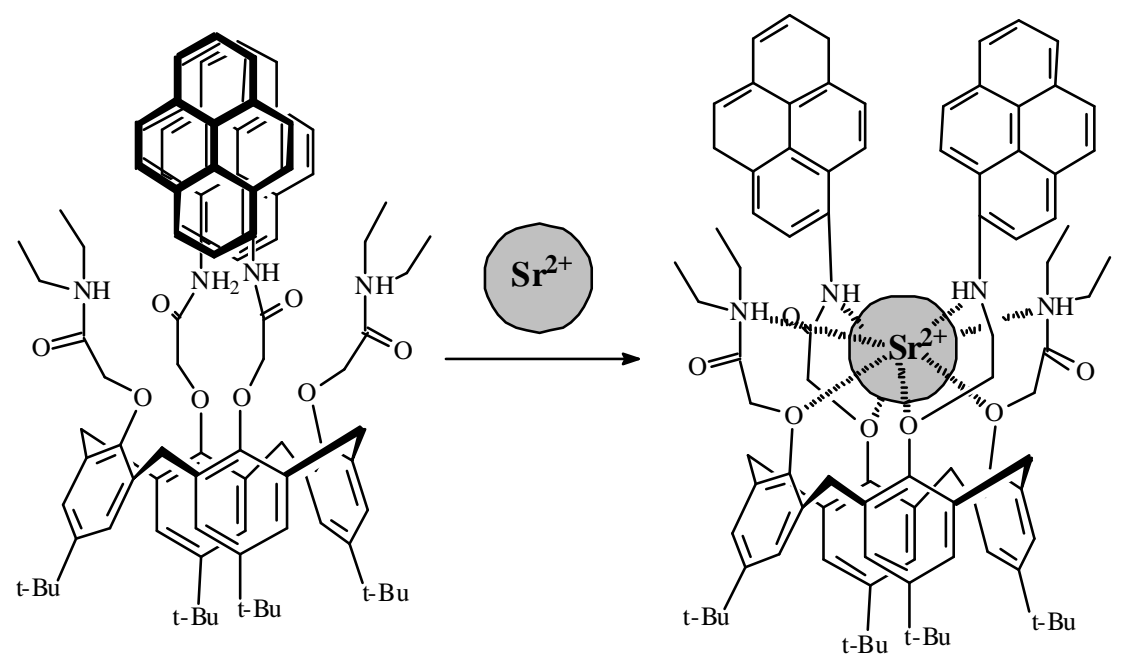

The fluorescence profile of this probe, attributable to the pyrene groups, changes upon complexation with $\mathrm{Sr}^{2+}$. In the absence of $\mathrm{Sr}^{2+}$, both monomer (370nm) and excimer (440nm) fluorescence of pyrene are observed. Upon addition of $\mathrm{Sr}^{2+}$ to a solution of the sensor molecule, the fluorescence of the excimer decreases while the monomer fluorescence peak increases indicating the pyrene groups are further apart. The ratio of the relative fluorescence intensity of pyrene monomer/excimer emission $\left(\mathrm{I}_{\mathrm{M}} / \mathrm{I}_{\mathrm{E}}\right)$ of PyCalix upon addition of $\mathrm{Sr}^{2+}$ is shown in the 
figure below. The $\mathrm{I}_{\mathrm{M}} / \mathrm{I}_{\mathrm{E}}$ ratio changed from 2.5 to 12 following the addition of sufficient $\mathrm{Sr}^{2+}$ to make a $10^{-5} \mathrm{M}$ solution. Unfortunately the ligand shows little discrimination

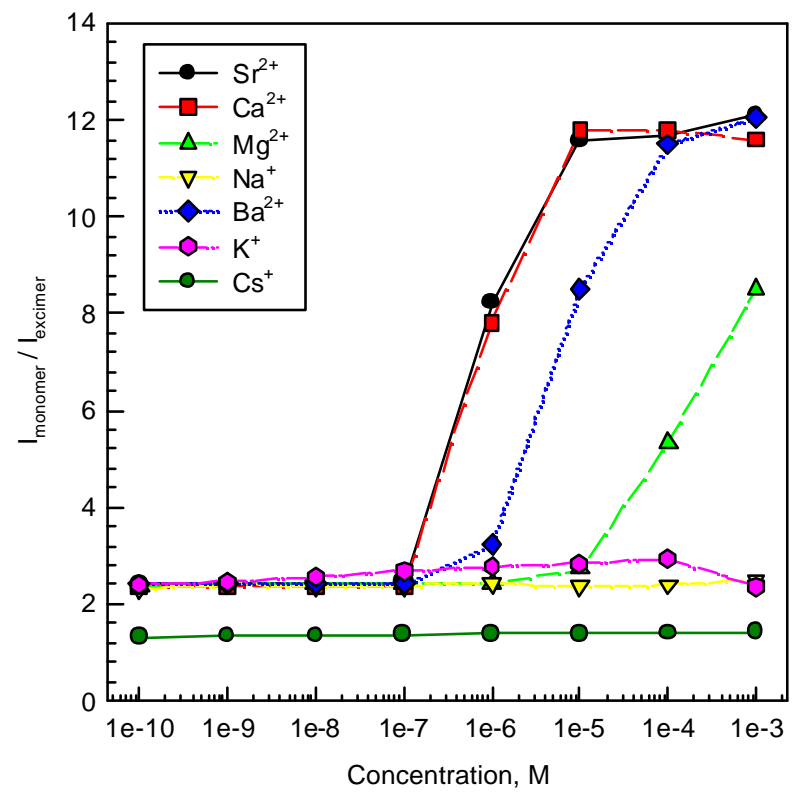
between $\mathrm{Sr}^{2+}$ and $\mathrm{Ca}^{2+}$ or $\mathrm{Ba}^{2+}$, although it effectively rejects $\mathrm{Cs}^{+}$. It is encouraging that analogous results were obtained when the ligand is embedded in a cellulose triacetate film, suggesting these results will translate to a polymer film for use with fiber optics.

The calix[4]arene-benzocrown-6ether derivative with a cyanoanthracene fluorophore had a large change in fluorescence upon complexation of $\mathrm{Cs}^{+}$ in solution, but it did not translate well to a polymer inclusion membrane film. In a search for fluorophores that are less sensitive to the medium, we are preparing the series of ligands shown in the diagram below. The crown compounds use two different isomers of coumarin as the fluorescent reporter, and the ether will be selective for $\mathrm{Cs}^{+}$while the amine derivatives should show selectivity for $\mathrm{Sr}^{2+}$.

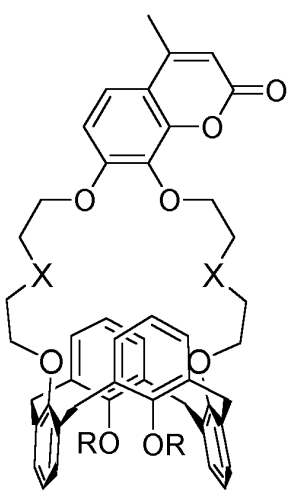

$\mathrm{R}=$ benzyl, propyl

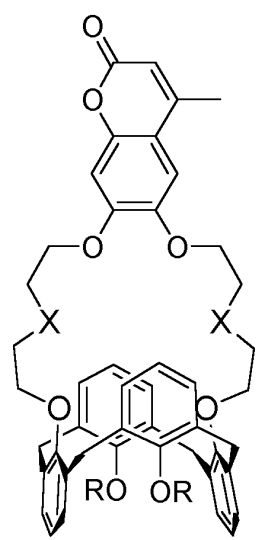

$\mathrm{R}=$ benzyl, propyl

\section{Planned Activities}

The objective of our investigations at the end of this project is to make arrays of cantilever sensors and fiber optic sensors for $\mathrm{Cs}^{+}, \mathrm{K}^{+}, \mathrm{Na}^{+}$, and $\mathrm{Sr}^{2+}$, based on known crown ether chemistry. We are planning to test these fiber optic and cantilever arrays first in cold waste simulant and then in actual Hanford tank waste. We will utilize literature formulations to add temperature and $\mathrm{OH}^{-}$sensor elements to the fiber optic and microcantilever arrays. The research in the next year will focus on improving stability in strong base solutions, and on gaining selectivity and sensitivity for the $\mathrm{Sr}^{2+}$ sensor elements.

\section{Information Access}

$\mathrm{X}=\mathrm{N}, \mathrm{O}$

Hao Xu , Xiaohe Xu, Reza Dabestani, Gilbert M. Brown, Lin Fan, Stephen Patton and Hai-Feng Ji, "Supramolecular Fluorescent Probes for the Detection of Mixed Alkali Metal Ions That Mimic the Function of Integrated Logic Gates," $J$. Chem. Soc. Perkin Trans 2, 2002, 636-643.. 